\title{
Generalized Configurations for the Synchronization System Based on Transfer Function Approach
}

\author{
Tuan Ngo \\ University of Texas at Austin \\ Tuyen $\mathrm{Vu}$ \\ Florida State University \\ Samet Biricik \\ Technological University Dublin, sbiricik@eul.edu.tr
}

Follow this and additional works at: https://arrow.tudublin.ie/engscheleart

Part of the Electrical and Computer Engineering Commons

\section{Recommended Citation}

T. Ngo, T. Vu and S. Biriciki, "Generalized Configurations for the Synchronization System Based on Transfer Function Approach," 2018 IEEE Power \& Energy Society General Meeting (PESGM), 2018, pp. 1-5, doi: 10.1109/PESGM.2018.8586297.

This Conference Paper is brought to you for free and open access by the School of Electrical and Electronic Engineering at ARROW@TU Dublin. It has been accepted for inclusion in Conference papers by an authorized administrator of ARROW@TU Dublin. For more information, please contact arrow.admin@tudublin.ie, aisling.coyne@tudublin.ie,gerard.connolly@tudublin.ie.

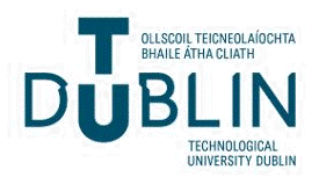




\title{
Generalized Configurations for the Synchronization System Based on Transfer Function Approach
}

\author{
Tuan $\mathrm{Ngo}^{1}$, Tuyen $\mathrm{Vu}^{2}$, and Samet Biriciki ${ }^{3}$ \\ ${ }^{1}$ Department of Electrical and Computer Engineering, The University of Texas at Austin, TX, USA \\ ${ }^{2}$ Center for Advanced Power Electronics, Florida State University, FL, USA \\ ${ }^{3}$ Department of Electrical and Computer Engineering, Dublin Institute of Technology, Dublin, Ireland
}

\begin{abstract}
This paper presents our research on deriving all the generalized forms for the grid synchronization system of an inverter in the grid-connected applications. The transfer function approach is introduced in order to obtain the generalized secondorder and third-order synchronization systems. This approach is based on the orthogonal property of two outputs from the second-order generalized-integrator (SOGI) technique that these two outputs are always $90^{\circ}$ difference in phase but only equal in magnitude at the grid frequency. The proposed approach then can provide the generalized forms of any high-order synchronization system, including the second-order and the third-order systems. It is interesting to see that the systems derived from this approach include all the previous synchronization systems in the literature. The simulation results have confirmed that these generalized systems are able to generate two perfectly orthogonal outputs to determine the grid information, i.e. voltage magnitude and phase angle. In addition, the generalized third-order system shows its excellent performance in removing harmonics under the distorted grid condition, and thus its ability to accurately detect the grid information.
\end{abstract}

Index Terms-Grid synchronization, second-order generalizedintegrator, third-order synchronization system, distorted grid.

\section{INTRODUCTION}

The second-order generalized-integrator (SOGI) technique was introduced and widely implemented in the grid-connected inverter applications [1]. This technique utilizes two integrators to form an oscillator in order to generate two orthogonal output signals. From these output signals, the grid information such as voltage magnitude and phase angle can be obtained. Due to its simplicity and robustness, this technique is preferred in the grid synchronization system of an inverter. However, one major drawback of this technique is that it cannot operate properly under distorted grid conditions due to the low attenuation capability of a second-order system. A possible solution is using multiple SOGIs in which many SOGI blocks are arranged in parallel to detect the fundamental voltage waveform and the harmonics [2], [3]. The harmonics are then used to cancel the harmonics in the input and thus results in sinusoidal outptus. This method, however, requires the information of all harmonic contents in the input voltage, and also needs many SOGI blocks arranged in parallel to eliminate harmonics and compute the fundamental waveform.

Few attempts trying to improve the performance of grid synchronization under distorted grid conditions have been proposed. The most common methods are adding another filter such as a compensator [4], [5] or a digital filter [6], [7] to remove harmonics. Another approach involve increasing the SOGI system to a third-order one. This work can be simply achieved by adding an integrator to the conventional SOGI structure. Due to higher attenuation capability, the third-order systems have better behaviors to harmonic distortions. For example, in [8], the complex vector is employed to derive the grid synchronization configuration. Since the complex-vector pre-filter is adopted, the system is able to lock its outputs at the fundamental frequency with sinusoidal waveforms. Another similar work is presented in [9]. An integrator is employed to the conventional SOGI to filter a DC offset from grid voltages. This third-order configuration can simply remove offset in comparison to the method proposed in [10]. More importantly, this method can filter harmonics in unbalanced voltages for grid synchronization. Our motivation in this paper is to search all the generalized second-order and third-order systems that can be utilized for the grid synchronization under various grid conditions. We introduce the transfer function approach in order to obtain all these generalized systems.

This paper begins in Section II by reviewing the operation principle of the second-order generalized-integrator through the transfer functions and its Bode plots. The proposed transfer function method is then introduced to obtain the generalized forms of a second-order and a third-order systems for grid synchronization. This method is based on the observation that the two outputs of a synchronization system are always orthogonal and are only equal in magnitude at the grid frequency. Many configurations for grid synchronization are derived, and it is interesting to see that this proposed method includes all the previous synchronization configurations in the literature. Section III presents the performance the derived second-order and third-order systems under different scenarios: a purely sinusoidal input and a distorted input. Simulation results show that the two derived systems are able to generate two perfectly orthogonal outputs from the input. In other words, these systems can be used for the grid synchronization. The performance of two derived systems are also verified with a distorted input. The results show that the second-order system cannot completely the harmonics, and thus results in two distorted outputs. The third-order system, on the other hand, is able to reject the harmonics. The outputs from this system are therefore perfectly sinusoidal and orthogonal. Finally, Section IV delivers remark conclusions for the applications of the transfer function approach for the grid synchronization system. 


\section{GRID SYNCHRONIZATION CONFIGURATIONS BASED ON The TRANSFER Function APPROACH}

This section first reviews the operating principle of secondorder generalized-integrator through its transfer functions and Bode plots. The transfer function approach for second-order and third-order systems are then presented to obtain the generalized grid synchronization configurations.

\section{A. Review of the second-order generalized-integrator}

The second-order generalized-integrator (SOGI) method was introduced for the grid-connected inverter applications to synchronize with the power grid [1]. This method utilizes two integrators to form a second-order oscillator as shown in Fig. 1. The SOGI generates two outputs, $v^{\prime}$ and $q v^{\prime}$, which are purely sine and cosine signals to calculate the rms value and the phase angle of the sinusoidal input $v_{i n}$.

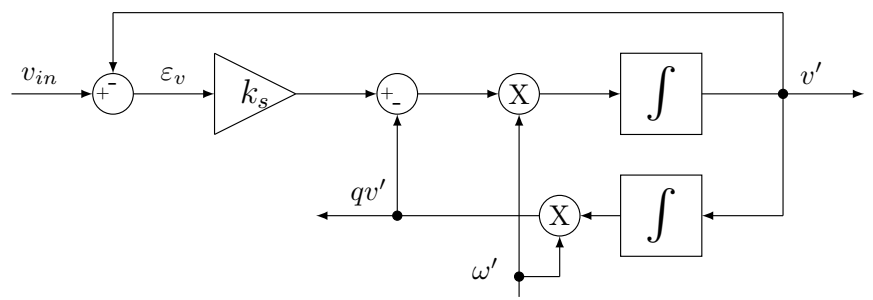

Fig. 1. A typical SOGI configuration for the grid synchronization.

The operating principles of SOGI can be explained from the transfer functions of two outputs [1] and from the Bode plots. Assuming $G_{V^{\prime}}(s)$ and $G_{Q V^{\prime}}(s)$ are the transfer functions of outputs $V^{\prime}(s)$ and $Q V^{\prime}(s)$ corresponding the input $V_{i n}(s)$, respectively, then

$$
\begin{aligned}
G_{V^{\prime}}(s) & =\frac{k_{s} \omega^{\prime} s}{s^{2}+k_{s} \omega^{\prime} s+\omega^{\prime 2}} \\
G_{Q V^{\prime}}(s) & =\frac{k_{s} \omega^{\prime 2}}{s^{2}+k_{s} \omega^{\prime} s+\omega^{\prime 2}}
\end{aligned}
$$

where $\omega^{\prime}$ is the central frequency, i.e. the power system frequency [11], and $k_{s}$ is a gain that can be changed for the system dynamics [1]. It is worthwhile to mention that the SOGI also behaves as an active filter oscillating at the frequency $\omega^{\prime}$ [1] and is able to remove the harmonics in case the input $v_{i n}$ is not purely sinusoidal. The Bode plot of these two transfer functions are visualized in Fig. 2. It can be seen that the magnitudes of two outputs are only equal at the central frequency $\omega^{\prime}$ while the phases are different in $90^{\circ}$ at any frequency which means the two outputs are always sine and cosine signals.

\section{B. The transfer function approach for second-order systems}

Validating the two transfer functions $G_{V^{\prime}}(s)$ and $G_{Q V^{\prime}}(s)$ at the central frequency $s=j \omega^{\prime}$, then

$$
\begin{aligned}
G_{V^{\prime}}\left(s=j \omega^{\prime}\right) & =\frac{k_{s} \omega^{\prime}\left(j \omega^{\prime}\right)}{\left(j \omega^{\prime}\right)^{2}+k_{s} \omega^{\prime}\left(j \omega^{\prime}\right)+\omega^{\prime 2}}=1 \\
G_{Q V^{\prime}}\left(s=j \omega^{\prime}\right) & =\frac{k_{s} \omega^{\prime 2}}{\left(j \omega^{\prime}\right)^{2}+k_{s} \omega^{\prime}\left(j \omega^{\prime}\right)+\omega^{\prime 2}}=-j
\end{aligned}
$$

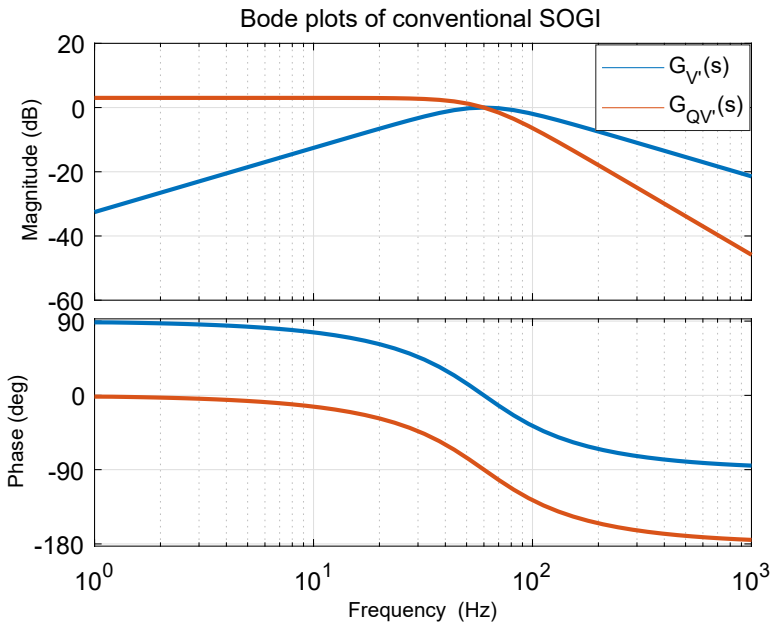

Fig. 2. Bode plots of $G_{V^{\prime}}(s)$ and $G_{Q V^{\prime}}(s)$.

The results from (3) and (4) explain for the sine and cosine signals in the SOGI if the input is a sinusoidal waveform. Note that the relation $G_{Q V^{\prime}}(s)=\left(\omega^{\prime} / s\right) \times G_{V^{\prime}}(s)$ is always held for a sine and a cosine functions. The generalized second-order forms for $G_{V^{\prime}}(s)$ and $G_{Q V^{\prime}}(s)$ can be given by

$$
\begin{aligned}
G_{V^{\prime}}(s) & =\frac{a_{0} s^{2}+a_{1} s+a_{2}}{s^{2}+b_{1} s+b_{2}} \\
G_{Q V^{\prime}}(s) & =\frac{\omega^{\prime}}{s} \times \frac{a_{0} s^{2}+a_{1} s+a_{2}}{s^{2}+b_{1} s+b_{2}}=\frac{\omega^{\prime}\left(a_{0} s^{2}+a_{1} s+a_{2}\right)}{s\left(s^{2}+b_{1} s+b_{2}\right)}
\end{aligned}
$$

A quick notice from (5) that if $a_{2} \neq 0$ then $G_{Q V^{\prime}}(s)$ is a third-order system while $G_{V^{\prime}}(s)$ is a second-order system. Since $G_{V^{\prime}}(s)$ and $G_{Q V^{\prime}}(s)$ are derived from one system, they should be the same denominator. In other words, $a_{2}$ should be equal to 0 . Evaluating $G_{V^{\prime}}(s)$ at the central frequency, $s=j \omega^{\prime}$, and set it to 1 then

$$
G_{V^{\prime}}\left(s=j \omega^{\prime}\right)=\frac{a_{0} s^{2}+a_{1} s}{s^{2}+b_{1} s+b_{2}}=\frac{-a_{0} \omega^{\prime 2}+a_{1} j \omega^{\prime}}{-\omega^{\prime 2}+b_{1} j \omega^{\prime}+b_{2}}=1
$$

By grouping real and imaginary parts from (6), the coefficients relation is: $b_{1}=a_{1}$ and $b_{2}=\left(1-a_{0}\right) \omega^{\prime 2}$. The generalized transfer functions are then

$$
\begin{aligned}
G_{V^{\prime}}(s) & =\frac{a_{0} s^{2}+a_{1} s}{s^{2}+a_{1} s+\left(1-a_{0}\right) \omega^{\prime 2}} \\
G_{Q V^{\prime}}(s) & =\frac{\omega^{\prime}\left(a_{0} s+a_{1}\right)}{s^{2}+a_{1} s+\left(1-a_{0}\right) \omega^{\prime 2}}
\end{aligned}
$$

Equations from (7) and (8) present the generalized forms of a second-order grid synchronization system. Fig. 3 shows the Bode plots of these two transfer functions with $a_{0}=0.5$ and $a_{1}=\sqrt{2} \omega^{\prime}$. As can be seen from that figure, the two outputs are only equal in magnitude at the central frequency. The phases of these outputs, however, are always $90^{\circ}$ difference. 
It is interesting to see that if $a_{0}=0$ and $a_{1}=k \omega^{\prime}$, then (7) and (8) becomes

$$
\begin{aligned}
G_{V^{\prime}}(s) & =\frac{k_{s} \omega^{\prime} s}{s^{2}+k_{s} \omega^{\prime} s+\omega^{\prime 2}} \\
G_{Q V^{\prime}}(s) & =\frac{k_{s} \omega^{\prime 2}}{s^{2}+k_{s} \omega^{\prime} s+\omega^{\prime 2}}
\end{aligned}
$$

which is nothing than the transfer functions of SOGI in the literature and was presented in the previous section. In other words, the equations in (7) and (8) provide the general forms of any second-order grid synchronization system.

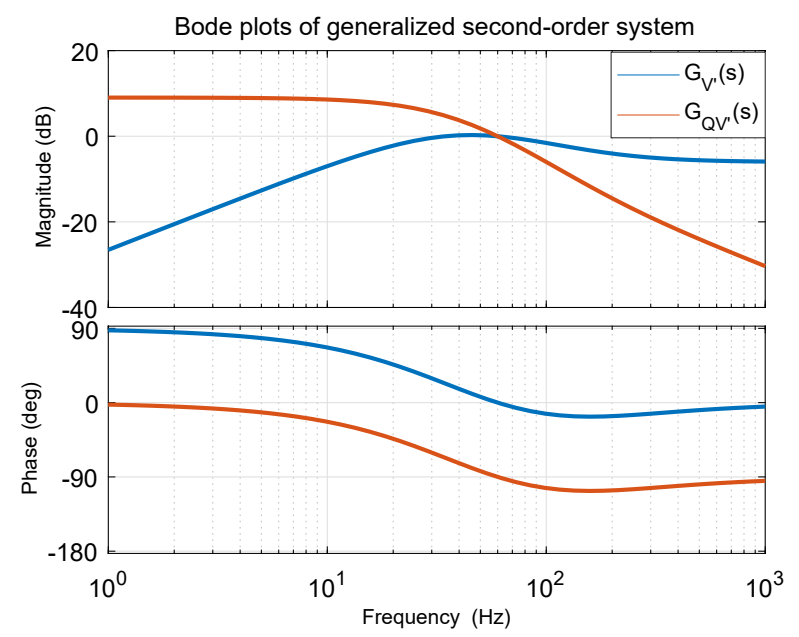

Fig. 3. Bode plots of the generalized second-order synchronization system.

\section{The transfer function approach for third-order systems}

The third-order systems have benefits over the second-order ones in terms of harmonic blocking [8], [9]. This section is thus to derive the generalized forms for the third-order system. Note that the third-order system still has two functions: 1) generating two orthogonal output signals, and 2) satisfying equations (3) and (4) for synchronization. It is worthwhile to note that once the transfer function $G_{V^{\prime}}(s)$ is determined, $G_{Q V^{\prime}}(s)$ can be found by $G_{Q V^{\prime}}(s)=G_{V^{\prime}}(s) \times\left(\omega^{\prime} / s\right)$. Therefore, the first step is to find $G_{V^{\prime}}(s)$. Assuming the transfer function starts with the general formulation of a thirdorder system

$$
G_{V^{\prime}}(s)=\frac{N(s)}{D(s)}=\frac{b_{0} s^{3}+b_{1} s^{2}+b_{2} s+b_{3}}{s^{3}+a_{1} s^{2}+a_{2} s+a_{3}}
$$

For the sake of simplicity, the denominator of (11) is first evaluated at the central frequency, $\omega^{\prime}$. Denoting $k_{i}=\left(a_{2}-\right.$ $\left.\omega^{\prime 2}\right), k_{r}=\left(a_{1}-a_{3} / \omega^{\prime 2}\right)$, and $j^{2}=-1$, then at $s=j \omega^{\prime}$

$$
\begin{aligned}
D\left(s=j \omega^{\prime}\right) & =\left(j \omega^{\prime}\right)^{3}+a_{1}\left(j \omega^{\prime}\right)^{2}+a_{2}\left(j \omega^{\prime}\right)+a_{3} \\
& =\left(a_{3}-a_{1} \omega^{\prime 2}\right)+j \omega^{\prime}\left(a_{2}-\omega^{\prime 2}\right) \\
& =k_{r}\left(-\omega^{\prime 2}\right)+k_{i}\left(j \omega^{\prime}\right)=k_{r}\left(j \omega^{\prime}\right)^{2}+k_{i}\left(j \omega^{\prime}\right) \\
& \triangleq k_{r}(s)^{2}+k_{i}(s) \quad \text { for }\left(s=j \omega^{\prime}\right)
\end{aligned}
$$

From (12), the numerator of $G_{V^{\prime}}(s)$ can be simply selected as: $N(s)=k_{r} s^{2}+k_{i} s$. The transfer functions of the thirdorder synchronization system is thus given by

$$
\begin{aligned}
G_{V^{\prime}}(s) & =\frac{k_{r} s^{2}+k_{i} s}{s^{3}+a_{1} s^{2}+\left(\omega^{\prime 2}+k_{i}\right) s+\left(a_{1}-k_{r}\right) \omega^{\prime 2}} \\
G_{Q V^{\prime}}(s) & =\frac{\left(k_{r} s+k_{i}\right) \omega^{\prime}}{s^{3}+a_{1} s^{2}+\left(\omega^{\prime 2}+k_{i}\right) s+\left(a_{1}-k_{r}\right) \omega^{\prime 2}}(13)
\end{aligned}
$$

Fig. 4 shows the Bode plots of two transfer functions in (13) with $k_{r}=20, k_{i}=22214.41, a_{1}=444.29$. The system is stable at pole locations $s_{1}=-402.42, s_{2,3}=-20.94 \pm$ $j 321.90$ and able to synchronize at the central frequency $\omega^{\prime}$. The output signals are always orthogonal because the phase difference is $90^{\circ}$.

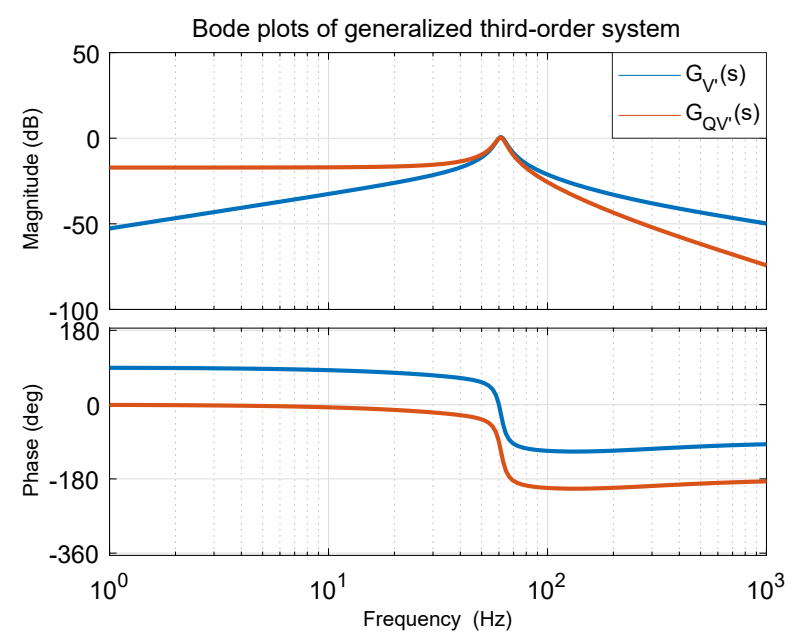

Fig. 4. Bode plots of third-order synchronization system.

The transfer functions in (13) can be simplified for some interesting cases: 1) $k_{r}=0$ and 2) $k_{i}=0$. These two cases can lead to configuration derivations based on SOGI structure in the literature [8], [9] for third-order synchronization systems.

Case 1: $k_{r}=0$ and the output transfer functions $G_{V^{\prime}}(s)$ and $G_{Q V^{\prime}}(s)$ are simplified as

$$
\begin{aligned}
G_{V^{\prime}}(s) & =\frac{k_{i} s}{s^{3}+a_{1} s^{2}+\left(\omega^{\prime 2}+k_{i}\right) s+a_{1} \omega^{\prime 2}} \\
G_{Q V^{\prime}}(s) & =\frac{k_{i} \omega^{\prime}}{s^{3}+a_{1} s^{2}+\left(\omega^{\prime 2}+k_{i}\right) s+a_{1} \omega^{\prime 2}}
\end{aligned}
$$

Case 2: $k_{i}=0$ and the output transfer functions $G_{V^{\prime}}(s)$ and $G_{Q V^{\prime}}(s)$ become

$$
\begin{aligned}
G_{V^{\prime}}(s) & =\frac{k_{r} s^{2}}{s^{3}+a_{1} s^{2}+\omega^{\prime 2} s+\left(a_{1}-k_{r}\right) \omega^{\prime 2}} \\
G_{Q V^{\prime}}(s) & =\frac{k_{r} \omega^{\prime} s}{s^{3}+a_{1} s^{2}+\omega^{\prime 2} s+\left(a_{1}-k_{r}\right) \omega^{\prime 2}}
\end{aligned}
$$

From the transfer function expressions in (13), (14), and (15), there are many configurations can be derived based on the state-space model. The most familiar systems are the controllable canonical form (CCF) and the observable canonical 
form (OCF) [12]. Fig. 5 shows an example of synchronization structure for (13) under the controllable canonical form.

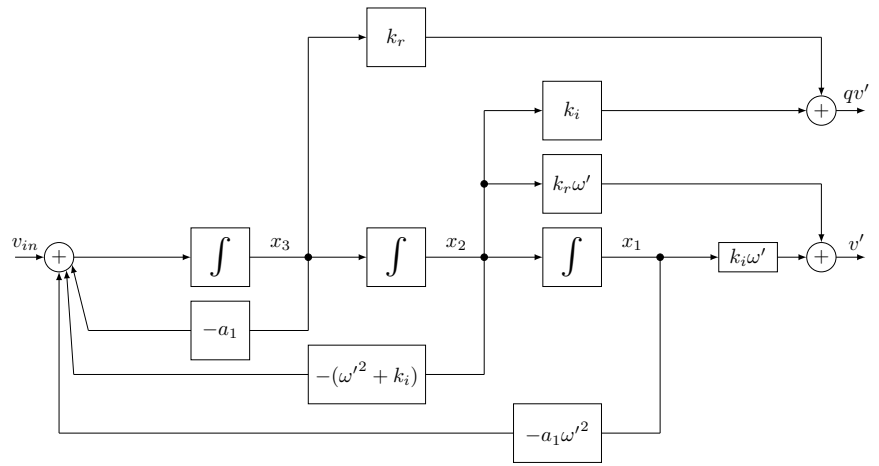

Fig. 5. A grid synchronization configuration based on CCF.

The third-order synchronization system can be also constructed based on a typical SOGI configuration in order to get benefits for the frequency controller. Denoting $a_{1}=k_{s} \omega^{\prime}$, $k_{i}=k_{a} k_{s} \omega^{\prime}$, and $k_{r}=k k_{s} \omega^{\prime}$, a state-space model for the system in (13) is given by

$\left[\begin{array}{c}\dot{x}_{1} \\ \dot{x}_{2} \\ \dot{x}_{3}\end{array}\right]=-\left[\begin{array}{ccc}0 & -1 & 0 \\ \omega^{\prime 2} & k_{s} \omega^{\prime} & (k-1) k_{s} \omega^{\prime} \\ \omega^{\prime 2} & \frac{k_{a}}{1-k} & 0\end{array}\right]\left[\begin{array}{l}x_{1} \\ x_{2} \\ x_{3}\end{array}\right]+\left[\begin{array}{c}0 \\ k k_{s} \omega^{\prime} \\ \frac{k_{a}}{1-k}\end{array}\right] V_{i n}$

The structure for (16) is shown in Fig. 6a based on the SOGI configuration. In addition, the configurations for (14) and (15) are investigated and presented in Fig. 6b and Fig. 6c, respectively. It is interesting to note that in case where $k_{r}=0$ and $k_{i}=0$, the configurations match with structures in [8], [9]. The detailed analyses can be found in these references.

\section{Simulation Results}

The generalized second-order and third-order systems are then modeled in MATLAB/SIMULINK to verify their synchronization capability. Note that for the third-order system in theory has $60 \mathrm{~dB}$ harmonic attenuation compared to $40 \mathrm{~dB}$ of that in the second-order system. The third-order system is thus expected to have better performance with the harmonics, and this functionality is also verified. The two systems are therefore tested at two different scenarios: with a sinusoidal input and with a distorted input.

\section{A. Performance with a sinusoidal input}

In this case, the input is a sinusoidal waveform as shown in Fig. 7a and is given by

$$
v_{\text {in }}(t)=120 \sqrt{2} \sin (2 \pi \times 60 \times t)
$$

The outputs of the generalized second-order and third-order systems are presented in Fig. 7b and Fig. 7c, respectively. As can be seen from these figures, the outputs $v^{\prime}(t)$ and $q v^{\prime}(t)$ are perfectly sinusoidal and equal in magnitudes. This means the two proposed systems can be used for grid synchronization. It is worthwhile to mention that the third-order system requires more time to reach the steady state compared to the secondorder system because it has higher order.

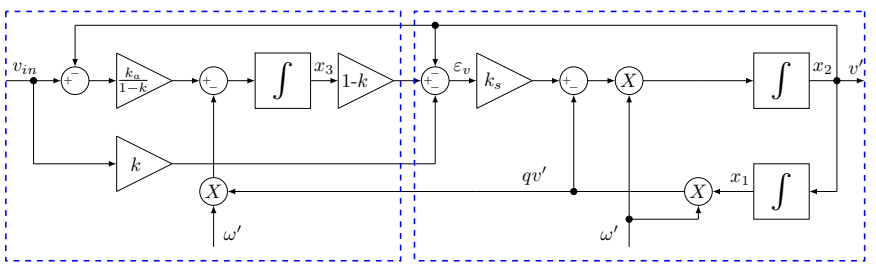

(a)

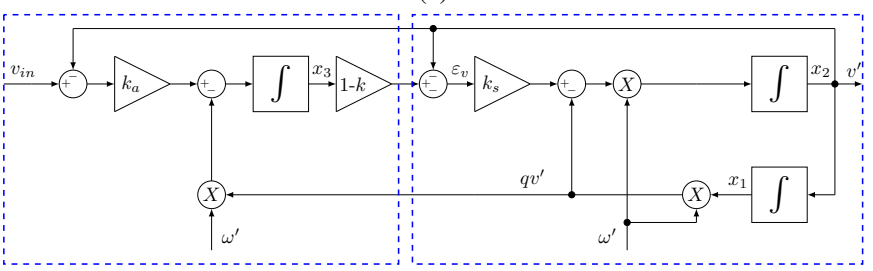

(b)

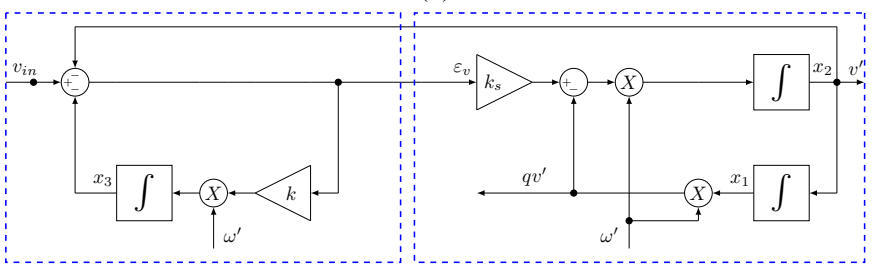

(c)

Fig. 6. Configurations based on SOGI: a) general case, b) $k_{r}=0$, and c) $\left(16_{i}\right)=0$.

\section{B. Performance with a distorted input}

In this scenario, the input voltage also contains the thirdorder and fifth-order harmonics as shown in Fig. 8a and is expressed as

$$
\begin{aligned}
v_{\text {in }}(t)= & 120 \sqrt{2} \sin (2 \pi \times 60 \times t)+20 \sqrt{2} \sin (2 \pi \times 180 \times t+\pi / 3) \\
& +5 \sqrt{2} \sin (2 \pi \times 300 \times t+\pi / 5)
\end{aligned}
$$

The performance of the generalized systems are shown in Fig. 8b and Fig. 8c. As expected, the second-order system cannot completely reject the harmonics and thus the outputs are not purely sinusoidal. The generalized third-order system, on the other hand, is able to generate two perfect sine and cosine waveforms. This result verifies the benefits of the thirdorder system over the second-order system in synchronization under harmonic conditions.

\section{CONCLusion}

In summary, this paper presents the research on grid synchronization based on the transfer function approach. By utilizing this method, the generalized forms of the secondorder and third-order systems are derived. These transfer function forms are more general and cover all the previous forms in the literature. Based on these transfer functions, many grid synchronization configurations have been constructed and verified in the simulation. The results confirm that the proposed second-order and third-order systems can be used for synchronization under normal and distorted grid conditions. 


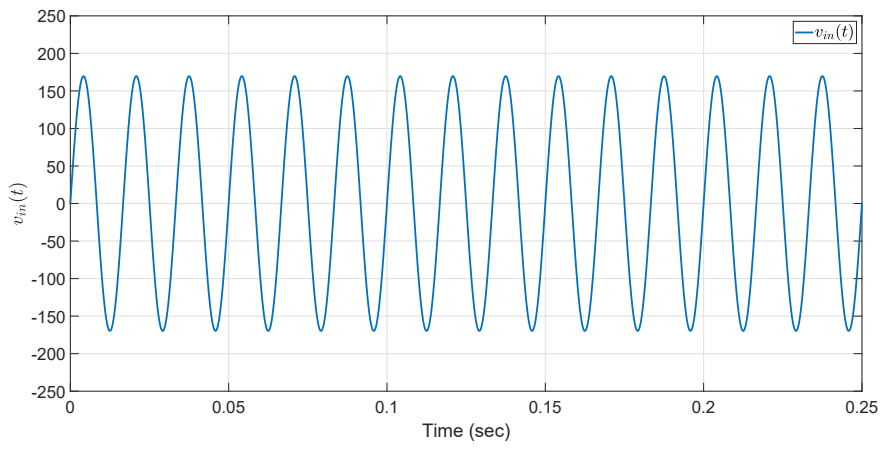

(a)

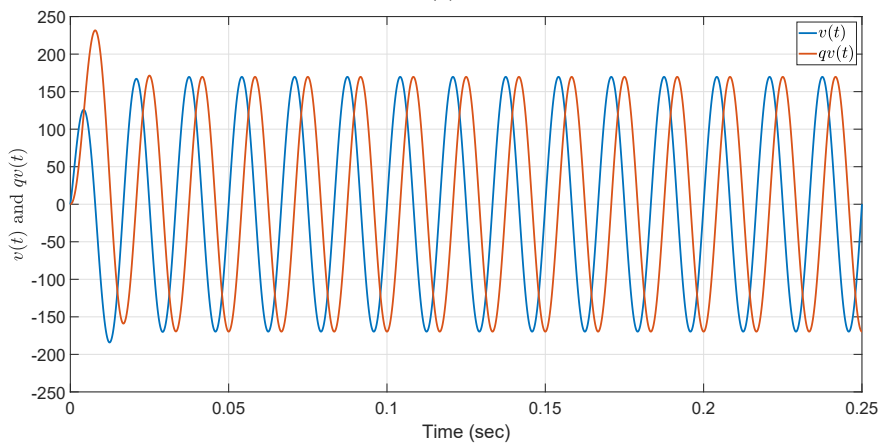

(b)

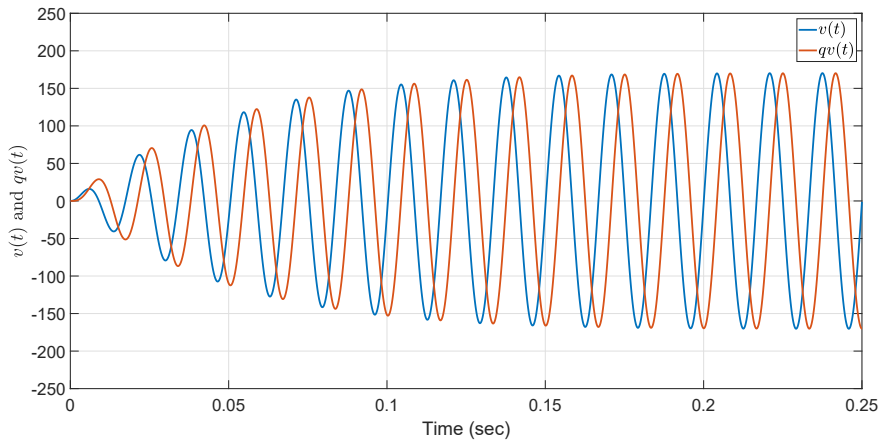

(c)

Fig. 7. Performance with a sinusoidal input voltage: a) the input voltage, b) the second-order system outputs, and c) the third-order system outputs.

\section{REFERENCES}

[1] R. Teodorescu, M. Liserre, and P. Rodriguez, Grid Converter for Photovoltaic and Wind Power Systems. Willey, 2011.

[2] P. Rodriguez, A. Luna, I. Candela, R. Teodorescu, and F. Blaabjerg, "Grid synchronization of power converters using multiple second order generalized integrators," in 34th IEEE IECON 2008., 2008, pp. 755-760.

[3] L. Hadjidemetriou, E. Kyriakides, Y. Yang, and F. Blaabjerg, "A synchronization method for single-phase grid-tied inverters," Power Electronics, IEEE Transactions on, vol. PP, no. 99, pp. 1-1, 2015.

[4] F. Freijedo, A. Yepes, O. Lpez, A. Vidal, and J. Doval-Gandoy, "Threephase plls with fast postfault retracking and steady-state rejection of voltage unbalance and harmonics by means of lead compensation," Power Electronics, IEEE Transactions on, vol. 26, no. 1, pp. 85-97, Jan 2011.

[5] F. Freijedo, J. Doval-Gandoy, O. Lopez, and E. Acha, "Tuning of phaselocked loops for power converters under distorted utility conditions," Industry Applications, IEEE Transactions on, vol. 45, no. 6, pp. 20392047, Nov 2009.

[6] F. Neves, H. de Souza, M. Cavalcanti, F. Bradaschia, and E. Bueno, "Digital filters for fast harmonic sequence component separation of unbalanced and distorted three-phase signals," Industrial Electronics, IEEE Transactions on, vol. 59, no. 10, pp. 3847-3859, Oct 2012.

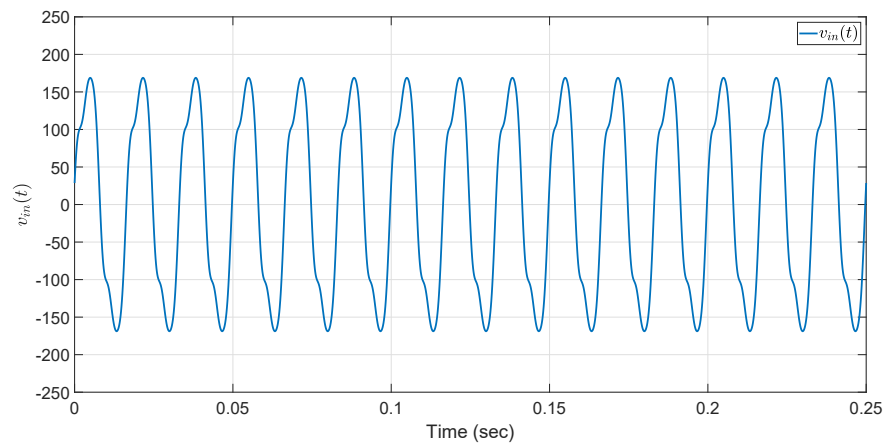

(a)

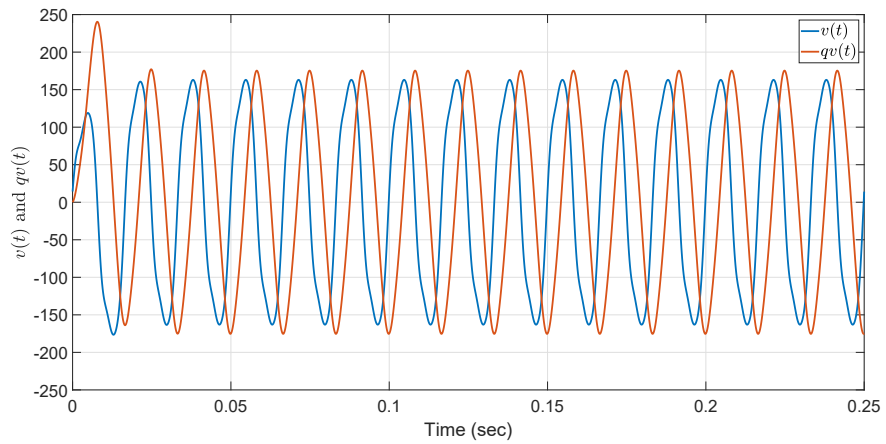

(b)

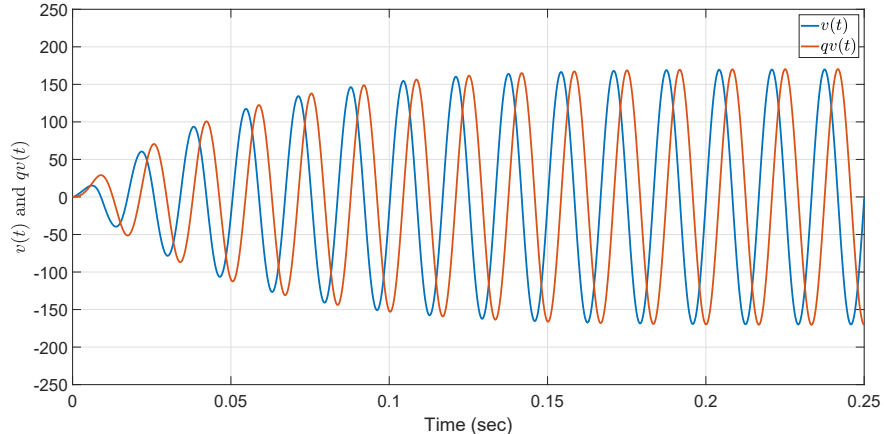

(c)

Fig. 8. Performance with a distorted input voltage: a) the input voltage, b) the second-order system outputs, and c) the third-order system outputs.

[7] X. Guo, W. Wu, and Z. Chen, "Multiple-complex coefficient-filterbased phase-locked loop and synchronization technique for three-phase grid-interfaced converters in distributed utility networks," Industrial Electronics, IEEE Transactions on, vol. 58, no. 4, pp. 1194-1204, April 2011.

[8] J. Li, J. Zhao, J. Wu, and P. ping Xu, "Improved dual second-order generalized integrator pll for grid synchronization under non-ideal grid voltages including dc offset," in Energy Conversion Congress and Exposition (ECCE), 2014 IEEE, Sept 2014, pp. 136-141.

[9] W. Li, X. Ruan, C. Bao, D. Pan, and X. Wang, "Grid synchronization systems of three-phase grid-connected power converters: A complexvector-filter perspective," Industrial Electronics, IEEE Transactions on, vol. 61, no. 4, pp. 1855-1870, April 2014.

[10] T. Ngo, Q. Nguyen, and S. Santoso, "Improving performance of singlephase sogi-fll under dc-offset voltage condition," in Industrial Electronics Society, IECON 2014 - 40th Annual Conference of the IEEE, Oct 2014, pp. $1537-1541$.

[11] P. Rodriguez, R. Teodorescu, I. Candela, A. Timbus, M. Liserre, and F. Blaabjerg, "New positive-sequence voltage detector for grid synchronization of power converters under faulty grid conditions," in 37th IEEE PESC '06., 2006, pp. 1-7.

[12] F. Golnaraghi and B. Kuo, Automatic Control Systems. Wiley, 2009. 